\title{
Study of the impact of clicks and murmurs on cardiac sounds S1 and S2 through bispectral analysis
}

\author{
Yettou Nour El Houda BAAKEK ${ }^{\mathrm{a}}$, Imane DEBBAL ${ }^{\mathrm{a}}$, Hidayat BOUdIS ${ }^{\mathrm{a}}$, Sidi Mohammed El Amine DeBBAL ${ }^{\mathrm{a},}$ \\ ${ }^{a}$ Genie Biomedical Laboratory (GBM), Faculty of Technology, University A.B.Belkaid-Tlemcen BP 119, Tlemcen; Algeria BP \\ 119 \\ *E-mail address: adebbal@yahoo.fr
}

\begin{abstract}
This paper presents a study of the impact of clicks, and murmurs on cardiac sound S1, and S2, and the measure of severity degree through synchronization degree between frequencies, using bispectral analysis. The algorithm is applied on three groups of Phonocardiogram (PCG) signal: group A represents PCG signals having a morphology similar to that of the normal PCG signal without click or murmur, group B represents PCG signals with a click (reduced murmur), and group C represent PCG signals with murmurs.

The proposed algorithm permits us to evaluate and quantify the relationship between the two sounds S1 and S2 on one hand and between the two sounds, click and murmur on the other hand. The obtained results show that the clicks and murmurs can affect both the heart sounds, and vice versa. This study shows that the heart works in perfect harmony and that the frequencies of sounds S1, S2, clicks, and murmurs are not accidentally generated; but they are generated by the same generator system. It might also suggest that one of the obtained frequencies causes the others.

The proposed algorithm permits us also to determine the synchronization degree. It shows high values in group C; indicating high severity degrees, low values for group B, and zero in group A.

The algorithm is compared to Short-Time Fourier Transform (STFT) and continuous wavelet transform (CWT) analysis. Although the STFT can provide correctly the time, it can't distinguish between the internal components of sounds S1 and S2, which are successfully determined by CWT, which, in turn, cannot find the relationship between them.

The algorithm was also evaluated and compared to the energetic ratio. the obtained results show very satisfactory results and very good discrimination between the three groups.

We can conclude that the three algorithms (STFT, CWT, and bispectral analysis) are complementary to facilitate a good approach and to better understand the cardiac sounds
\end{abstract}

Key words: sound S1; sound S2; click, murmur; impact; bispectral; analysis, pathology; severity.

\section{Introduction}

The phonocardiogram (PCG) (Figure 1) is one of the most tools used for analyzing the mechanical activities of the heart, and for the diagnosis of abnormal heartbeat sounds such as murmur. PCG signal can describe with great precision its different components such as heart sounds: S1, S2, clicks, and murmurs.

When a healthy heartbeat, it makes a "lub-dub" sound. The first heart sound "lub", also known as S1 is caused by the closing of atrioventricular (AV) valves after the atria have pumped blood onto the ventricles (Figure 2).

The second heart sound "dub", or S2 originates from the closing of the aortic and pulmonary valves, right after the ventricles have ejected the blood. The time interval between S1 and S2 is when the ventricles contract, called Systole. The interval between $\mathrm{S} 2$ and next $\mathrm{S} 1$ is when the ventricles relax and are filled with blood, called diastole. Diastole is longer than systole.

S1 is caused by the closure of mitral and tricuspid valves at the start of systole. The second heart sound S2, is caused by the closure of the aortic and pulmonic valves, marking the end of systole. $^{1,2}$

According to the literature, there are two major components of each sound. ${ }^{2,3}$ The components for the sound S1 are M1 and T1, one due to closure of the Mitral valve and the other due of the closure of the Tricuspid valve. Inspiration delays the closure of the tricuspid valve in a normal person, due to increased venous return, thereby enhancing the splitting of the S1 sound. This delays the closure of the tricuspid valve, resulting in a split $\mathrm{S} 1$ sound, and $\mathrm{M} 1$ and $\mathrm{T} 1$ are separated by a relatively short interval, however a split S1 can also be heard in the setting of a right bundle branch block (RBBB). 
For the second sound S2, one sound is due to the closure of the aortic valve (A2) and the other sound is due to the closure of the pulmonary valve $(\mathrm{P} 2){ }^{2,4,5}$ Specifically, during expiration, A2 and P2 are separated by a relatively short interval typically less than 30 ms. ${ }^{6}$ It is heard when aortic and pulmonic valves close at slightly different times.

However, there are two other kinds of heart sound which are: ejection clicks, and murmurs. Ejection clicks are high-pitched sounds that occur at the moment of maximal opening of the aortic or pulmonary valves. They are heard just after the first heart sound.

Heart murmurs are whooshing sounds produced by the turbulent flow of blood. Murmurs are diagnosed based on the time they occur in the cardiac cycle, their changes in intensity, over time, and the auscultation site where they are best heard.

PCG signal compared to other investigative techniques provides information in real-time with high resolution. In addition, from PCG signal some important parameters can be extracted such as: amplitude, frequency, number of components of the signal in each cycle, the intensity, the duration, the location, and the different shapes of each sound which are considered as indicators of diseases under study.

On the other hand, this kind of signal shows significant random and nonlinear properties, especially in the murmur sounds. Many studies have been carried out, on the behavior of this kind of signal. Some of these studies are discussed below.

Yaseen et $\mathrm{al}^{7}$ used Artificial Neural Networks (ANN) to detect and classify heart diseases. They extracted time domain and frequency domain features such as energy, mean, variance and Mel Frequency Cepstral Coefficients (MFCC). various heart sound samples are classified using Support Vector Machine (SVM), K Nearest Neighbour (KNN), Bayesian and Gaussian Mixture Model (GMM) Classifiers.

Mohamed Boussaa et $\mathrm{al}^{8}$ describe in detail a medical assistance system that can detect and distinguish between normal and abnormal heart signals. Their system combines firstly between the MFCC as a descriptor of the cardiac signal and the artificial neural network-type MLP as a classifier and secondly between the discrete wavelet descriptor, statistical operations as reducing the characteristic vector of the dimensions extracted and the same type of classifier.

Maciej Rosół et $\mathrm{al}^{9}$ present a low-cost system of location and classification of heart sounds in $\mathrm{S} 1$ and $\mathrm{S} 2$ based on a singlechip microcontroller and MEMS microphone. They also used the temporal, frequency analysis, autocorrelation function, and time-frequency to analyze diastole and systole heart cycles.

However, all these methods can't give us a relationship between the different frequencies; on the contrary, the bispectral analysis can provide this relationship, and can distinguish between them.

This study is a complementary study to that which was made by Debbal et $\mathrm{al}^{2}$. they found that the continuous wavelet transform is the most successful technique for the analysis of the internal components of these sounds. The continuous wavelet transform allows the best representation of the internal components (M1, T1, A2 and P2) and the measure of the time delay between these components. However, the STFT can provide correctly the time but the components M1 and T1 of the sound $\mathrm{S} 1$ and the components $\mathrm{A} 2$ and $\mathrm{P} 2$ of the second sound are not obviously detected and not accurately represented. For more details see reference 2.

In this work, our contribution is to find the relationship between the different PCG sounds (S1, S2, Click, and murmurs) on one hand and between the internal components of each sound on the other hand. In addition, the proposed algorithm permits us to determine a new parameter that corresponds to the synchronization degree between the frequencies using bispectral analysis. This parameter can give us information about the severity degree of the pathology. Finally, the work is evaluated and finished by a comparison between bispectral analysis, CWT and energetic ratio.

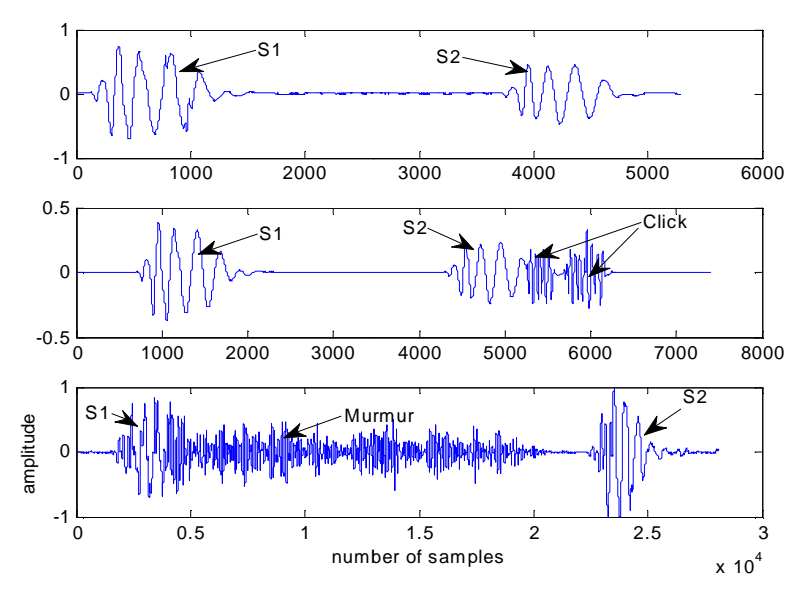

Figure 1. PCG signals used

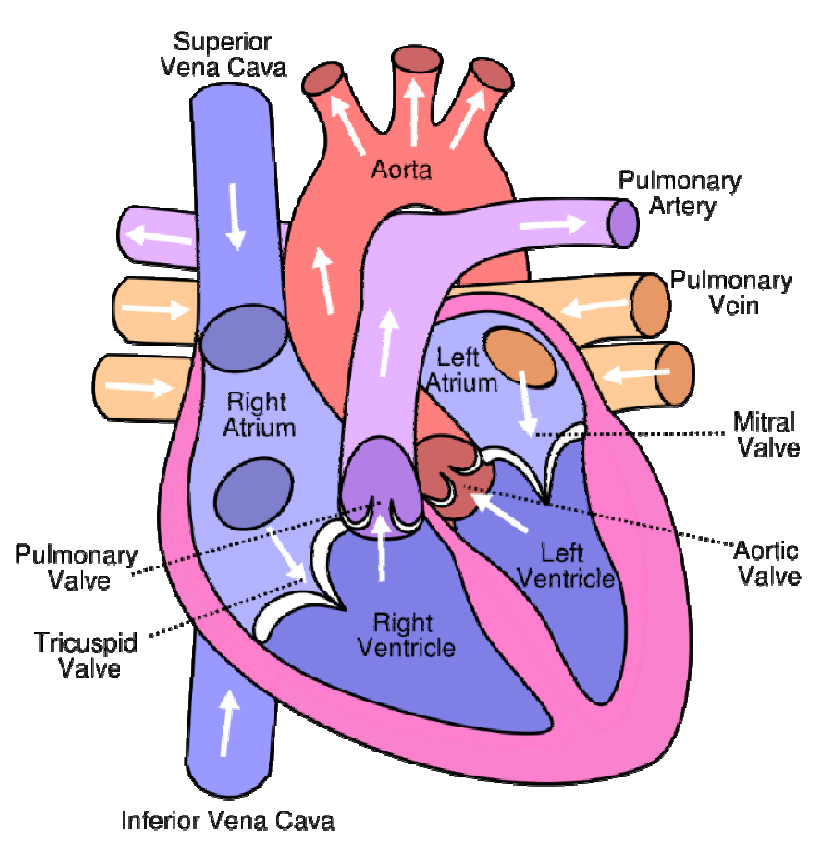

Figure 2. Human heart valves 


\section{Materials and Methods}

\section{Database}

This database consists of 11 different cases of PCG signals (Table 1), taken from three different databases, ${ }^{10-12}$ with different degrees of heart pathological severity. Each database has its own sampling frequency (see Table 1).

Heart sounds are auscultated at four different sites on the chest wall which corresponding to the location of blood flow as it passes through the aortic, pulmonic, tricuspid, and mitral valves, respectively. The diagnosis of the presence or absence of clicks and murmurs was taken from the source database. The severity of clicks and murmurs depends on the location of auscultation, the quality or the intensity, and their timing. We have grouped and classified the signals into three groups according to their pathological severity based on the segmentation of sounds and heart murmurs (Figure 2). ${ }^{13-16}$ Group A corresponding to PCG signals having a morphology similar to that of the normal PCG signal without click or murmur: " $n$ " normal heartbeat cardiac sound, "coa" coarctation of the aorta, and "IM" Innocent murmur.

Group B corresponding to PCG signals with a click (reduced murmur), it contains five PCG signals: "EC" Ejection click, "AG" Aortic Gallop, "EC1" Ejection click, "MP" systolic Mitral Prolapse, and "LS" Late systolic.

Group C corresponding to PCG signals with murmurs it contains three PCG signals: "Mr" Mitral regurgitation, "ar" aortic regurgitation, and "tr" Tricuspid regurgitation.

\section{Preprocessing}

Usually, PCG signals after acquisition are disturbed by various noises. One of the first operations is therefore to apply a filter that eliminates these disturbances. It is therefore preferable to filter this signal as a first step and before any analysis. The filter used is the discrete wavelet transform. For a better analysis, the choice of the mother wavelet is more important. Therefore, in this work, the most suitable analyzing wavelet for performing this filtering is: the 7th level Daubechies wavelet (db7). This latter is the most suitable for analyzing normal and pathological PCGs signals.

\section{Bispectral analysis}

A bispectral analysis is an advanced signal processing technique that quantifies quadratic nonlinearities among the components of a signal. ${ }^{17-19}$ It is defined as the Fourier transform of the third cumulant. Considering that the Fourier transform of the second-order cumulant (the auto-correlation function) is known by the power spectral density (PSD). The Hanning window length of 256 was used. The bi-spectral is defined by Equation 1.

$B\left(f_{1}, f_{2}\right)=E\left[X\left(f_{1}\right) X\left(f_{1}\right) X^{*}\left(f_{1}+f_{2}\right)\right]$

Eq. 1

Where $X(\mathrm{f})$ is the discrete Fourier transform (DFT) of (nT).

$\mathrm{X}^{*}(\mathrm{nT})$ is the conjugate of $\mathrm{x}(\mathrm{nT})$,

$E[$.$] : is the operator of mathematical expectation.$

$f 1+f 2$ : is the frequency of the harmonic component

Table 1. Description of the used database.

\begin{tabular}{|c|c|c|c|c|c|}
\hline Group & Record & $\begin{array}{c}\text { Sampling } \\
\text { Frequency }(\mathbf{H z})\end{array}$ & Signal length & $\begin{array}{c}\text { Cycles } \\
\text { number }\end{array}$ & Description of the pathology \\
\hline \multirow{3}{*}{ A } & $\mathrm{n}$ & 11025 & 49400 & 6 & Normal case \\
\hline & coa & 8012 & 11962 & 2 & $\begin{array}{l}\text { Coarctation of the aorta is a birth defect in a part of the aorta, the tube that } \\
\text { carries oxygen-rich blood to the body, is narrower than usual. It forces the heart } \\
\text { to pump harder to get blood through the aorta and on to the rest of the body. }\end{array}$ \\
\hline & IM & 8012 & 32465 & 5 & $\begin{array}{l}\text { An Innocent heart Murmur does not cause heart problems. It is often related to } \\
\text { a temporary condition, causing high blood flow through the heart's valves. }\end{array}$ \\
\hline \multirow{4}{*}{$\mathrm{B}$} & $\mathrm{EC}, \mathrm{EC} 1$ & 11025 & 54672 & 6,7 & $\begin{array}{l}\text { Ejection Clicks are high-pitched sounds which are heard just after the first heart } \\
\text { sound. They occur in the presence of a dilated aorta or pulmonary artery or in } \\
\text { the presence of a bicuspid or flexible stenotic aortic or pulmonary valve. }\end{array}$ \\
\hline & AG & 11025 & 52878 & 6 & $\begin{array}{l}\text { Aortic Gallop is an abnormal rhythm of the heart on auscultation. It contains } \\
\text { another sound, called S3 or S4, dependent upon where in the cycle this added } \\
\text { sound comes. }\end{array}$ \\
\hline & MP & 11025 & 54381 & 6 & $\begin{array}{l}\text { Mitral Prolapse is a disorder, usually benign, of the functioning of the mitral } \\
\text { valve and may be responsible for more or less severe mitral insufficiency. it is } \\
\text { characterized by the displacement of an abnormally thickened mitral valve } \\
\text { leaflet into the left atrium during systole. It is the primary form of myxomatous } \\
\text { degeneration of the valve. }\end{array}$ \\
\hline & LS & 11025 & 57024 & 6 & $\begin{array}{c}\text { Late Systolic is a sharp, clicking sound heard during cardiac late systole. It } \\
\text { signifies mitral insufficiency, may also be due to pleuropeicardial adhesions or } \\
\text { other extracardiac mechanisms }\end{array}$ \\
\hline \multirow{3}{*}{$\mathrm{C}$} & $\mathrm{Mr}$ & 44100 & 329728 & 9 & $\begin{array}{l}\text { Mitral Regurgitation It is one of the most common diseases among heart valve } \\
\text { pathologies. in this case, the heart's mitral valve doesn't close tightly, causing a } \\
\text { backflow of blood from the left ventricle into the left atrium during the phase of } \\
\text { expulsion of the blood (systole), instead of going into the aorta. }\end{array}$ \\
\hline & ar & 44100 & 586530 & 14 & $\begin{array}{c}\text { Aortic Regurgitation, is a heart valve disease corresponding to a failure to } \\
\text { check the aortic valves of the heart which causes retrograde blood flow during } \\
\text { diastole, from the aorta to the left ventricle }\end{array}$ \\
\hline & $\operatorname{tr}$ & 44100 & 200112 & 8 & $\begin{array}{l}\text { TRicuspid insufficiency is an impairment of the tricuspid valve allowing blood } \\
\text { to flow back from the right ventricle to the right atrium. }\end{array}$ \\
\hline
\end{tabular}


Since the correlation function is an even function, and its FT gives a symmetry spectrum (i.e. the spectrum repeats twice "mirror effect"), we find that the bi-spectrum which represents the FT of the tri-correlation function, repeats itself four times. It is therefore enough to calculate the spectrum for the frequencies that lie in the non-redundant region $\Omega$ as it is illustrated in Figure 3. For more details see ${ }^{20}$.

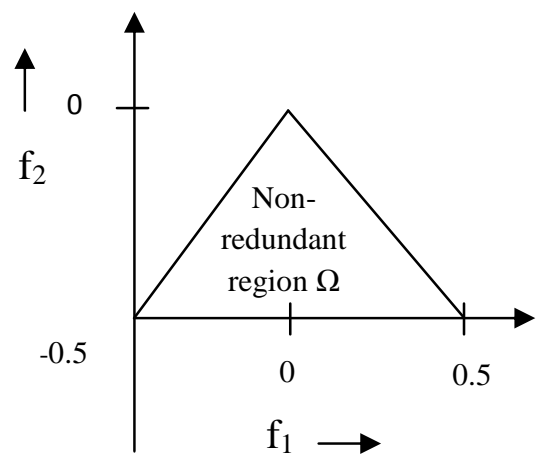

Figure 3. The non-redundant region for the calculation of bispectral frequencies. ${ }^{11}$

\section{Synchronization degree}

The synchronization degree is defined as the ratio between the number of harmonics and all the sinusoids number of the bispectrum. We must indicate here that the number of harmonic and the sinusoids must be calculating $\mathrm{n}$ times if it is generated $\mathrm{n}$ times.

Syn $=\frac{\sum_{k=1}^{n-1} f_{k}}{\sum_{i=1}^{n} f_{i k}}$

$f_{k}=f_{i}+f_{j}$

$i=1, \ldots, n$

$j=1, \ldots, n$

\section{Results and discussion}

The bispectral algorithm has been implemented and applied on three sets of PCG signals: group A corresponding to normal cases without click or murmur, group B corresponding to PCG signals with a click, and group C corresponding to PCG signals with murmurs. Heart sounds are auscultated at four different sites on the chest wall which corresponding to the location of blood flow as it passes through the aortic, pulmonic, tricuspid, and mitral valves, respectively. The obtained results are illustrated in Figures $\mathbf{4}$ to $\mathbf{1 2}$ and resumed in Tables $\mathbf{2}$ and $\mathbf{3}$.

\section{Group A}

The bispectra of the sound S1 and S2 of the record (coa.wav) in group A are illustrated in Figures $\mathbf{4}$ and $\mathbf{5}$ respectively. The color represents the relative variation of the amplitude of the bispectrum. The red color indicates the strongest increase, and the blue line indicates the greatest decrease.

The analysis of the internal components of heart sounds (M1 and T1 for S1: A2 and P2 for S2) makes it possible, through the measurements obtained and the variation of the parameters studied, to know the operating state of the corresponding valves (mitral (M1), tricuspid (T1), aortic (A2) and pulmonary (P2) which can help the clinician in establishing the medical diagnosis or the surgeon in the case of valve transplantation.

The bispectra show a predominance of two peaks in each sound: (M1 and T1) in sound S1, and (A2 and P2) in sound S2.

The peak M1 (Figure 4, Table 2) represents only one frequency, it is around $(179,43,179.43) \mathrm{Hz}$, however $\mathrm{T} 1$ is around $(179,43,358.86) \mathrm{Hz}$. As we remark the frequency $353.86 \mathrm{~Hz}$ represents a quadratic interaction, it is the double of the M1 component frequency $(358.86=179.43 * 2)$. The components M1 and T1 of sound S1 are due to the closing of the mitral valve and the tricuspid valve respectively. According to this result, we can say that the frequency of the signal corresponding to the closing tricuspid valve is double the frequency of the signal corresponding to the closing mitral valve.

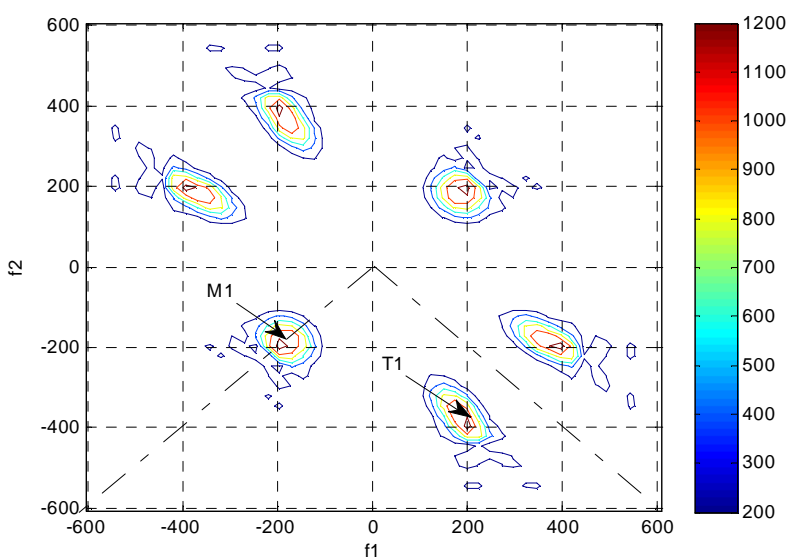

Figure 5. Bispectrum of S2 sound in normal case (Group A, record " $n$ ")
Figure 4. Bispectrum of $\mathrm{S1}$ sound in normal case (Group A, record "n") 
Similar results are obtained in the bispectrum of S2 (Figure 5). The peak A2 represents only one frequency, it is around $(239.24,239.24) \mathrm{Hz}$, however $\mathrm{P} 2$ is around $(239.24,478.48)$ $\mathrm{Hz}$, it represents a quadratic interaction $(478.48=239.24 * 2)$. According to this result, we can say that the frequency of the component A2 is double that of the component $\mathrm{P} 2$. This second sound S2 signal represents the end of the systole and the beginning of the diastole. However, the components A2 and P2 of $\mathrm{S} 2$ represent the closing of the aortic and pulmonary valves respectively. i.e. the frequency of the signal corresponding to the closing pulmonic valve is double the frequency of the signal corresponding to the closing aortic valve.

Similar results are obtained in the record "IM" in group A; however, in the signal " $n$ " which represents a normal case, we found that $\mathrm{A} 2$ equal to $\mathrm{M} 1$ and $\mathrm{P} 2$ equal to $\mathrm{T} 1$ (Table 2). According to this result, we can say that in the normal case the frequency of the signal corresponding to the closing of the mitral valve equal to the frequency of the signal corresponding to the closing of the aortic valve, and the frequency of the signal corresponding to the closing tricuspid valve equal of the frequency of the signal corresponding to the closing pulmonic valve. This result leads us to conclude that the bispectral analysis can distinguish between normal cases and pathologic cases even if it has a morphology similar to that of the normal PCG signal.

However, the synchronization degree and as is illustrated in Table 2, shows a zero value in all the cases of group A, because there is no harmonic component.

\section{Group B}

Similar results are also obtained in the bispectra of the sounds S1 and S2 (record EC), as it is illustrated in Figures 6 and 7 respectively. It shows a predominance of two peaks in each sound: (M1 and T1) in the sound S1, (A2 and P2) in the sound S2.

The peak M1 and A2 represent only one frequency, it is around $(273.36,273.36) \mathrm{Hz}$, however $\mathrm{T} 1$ and $\mathrm{P} 2$ are around (273.36, 546.72) Hz. As we remark, S1 and S2 are identical, and the frequency of $\mathrm{T} 1$ is twice the frequency $\mathrm{M} 1$, and the frequency of $\mathrm{P} 2$ is twice the frequency of $\mathrm{A} 2$.

However, the bispectrum of click in this signal shows also two peaks (Figure 8, Table 2), the first mr1 is around (820.08, $820.08) \mathrm{Hz}$, and the second $\mathrm{mr} 2$ is around $(820.08,1640.16)$ $\mathrm{Hz}$. According to this result, we can remark, that the frequency $1640.16 \mathrm{~Hz}$ is twice the frequency $820.8 \mathrm{~Hz}$. On other hand, the frequency $820.08 \mathrm{~Hz}$ results from the nonlinear interaction between the frequencies $546.72 \mathrm{~Hz}$ and $273.36 \mathrm{~Hz}$ which are found in the S1 and S2 bispectra; because it equals to the sum of two frequencies; which leads us to conclude that there is a nonlinear relationship between the click heart and the sounds S1 and S2, and that might suggest that the three sounds S1, S2, and click have the same generator system. It might also suggest that one of the obtained frequencies causes the others.

The synchronization degree between the different frequencies show a low value $(1 / 4=25 \%)$ (Table 2); because we have only one harmonic frequency (820.08) between four frequencies $(273.36,546.72,820.08,1640.16) \mathrm{Hz}$. Similar results are obtained in the other signals of group B, where we found low values that vary between $25 \%$ and $42 \%$.

Table 2. Summary table of the results obtained

\begin{tabular}{|c|c|c|c|c|c|}
\hline \multirow{2}{*}{ Group } & \multirow{2}{*}{ Record } & \multicolumn{2}{|c|}{ Heart Sound (S1/S2) } & \multirow{2}{*}{$\begin{array}{c}\text { Click } \\
\text { Cki }\end{array}$} & \multirow{2}{*}{$\begin{array}{c}\text { Murmur } \\
\text { MRi }\end{array}$} \\
\hline & & S1 (M1/T1) & $\mathrm{S} 2(\mathrm{~A} 2 / \mathrm{P2})$ & & \\
\hline \multirow{3}{*}{ A } & $\mathrm{n}$ & $\begin{array}{l}\text { M1 }(197.632,172.92) \\
\text { T1 }(197.632,370.53)\end{array}$ & $\begin{array}{l}\text { A2(197.632,172.928) } \\
\text { P2(197.632,370.53) }\end{array}$ & I & I \\
\hline & coa & $\begin{array}{l}\text { M1 }(179,43,179.43) \\
\text { T1 }(179,43,358.86)\end{array}$ & $\begin{array}{l}\text { A2 }(239.24,239.24) \\
\text { P2 }(239.24,478.48)\end{array}$ & l & l \\
\hline & $\mathrm{IM}$ & $\begin{array}{l}\text { M1 }(486.975,486.97) \\
\text { T1 }(486.975,973.95)\end{array}$ & $\begin{array}{l}\text { A2 }(324,65,324,65) \\
\text { P2(324.65, 649.3) }\end{array}$ & I & l \\
\hline \multirow{5}{*}{$\mathrm{B}$} & EC & $\begin{array}{l}\text { M1(273.36, 273.36) } \\
\text { T1 }(273.36,546.72)\end{array}$ & $\begin{array}{l}\text { A2(273.36, 273.36) } \\
\text { P2(273.36, 546.72) }\end{array}$ & $\begin{array}{l}\text { Ck1(820.08, 820.08) } \\
\text { Ck2(820.08,1640.16) }\end{array}$ & / \\
\hline & AG & $\begin{array}{c}\text { M1 }(264.39,264.39) \\
\text { T1 }(264.39,528.78)\end{array}$ & $\begin{array}{l}\text { A2 }(264.39,264.39) \\
\text { P2(264.39,528.78) }\end{array}$ & $\begin{array}{l}\text { Ck1 }(528.78,528.78) \\
\text { Ck2 }(528,78,793.17)\end{array}$ & / \\
\hline & $\mathrm{EC} 1$ & $\begin{array}{c}\text { M1(285.535,285,535) } \\
\text { T1 }(285.535,571.07)\end{array}$ & $\begin{array}{c}\text { A2(285.535,285,535) } \\
\text { P2(285.535,571.07) }\end{array}$ & $\begin{array}{l}\text { Ck1 }(815.715,815.715) \\
\text { Ck2 }(815.715,1631.43)\end{array}$ & / \\
\hline & MP & $\begin{array}{l}\text { M1(271.95, 271.905) } \\
\text { T1 }(271,905,534.81)\end{array}$ & $\begin{array}{c}\mathrm{A} 2(271.95,271.905) \\
\mathrm{P} 2\end{array}$ & $\begin{array}{l}\text { Ck1 }(815.715,815.715) \\
\text { Ck2 }(815,715,1631.43)\end{array}$ & I \\
\hline & LS & $\begin{array}{c}\mathrm{M} 1(285.12,285,12) \\
\mathrm{T} 1(285.12,570.24)\end{array}$ & $\begin{array}{l}\text { A2 }(285.12,285,12) \\
\text { P2(285.12,570.24) }\end{array}$ & Ck1 $(855.36,855.36)$ & l \\
\hline \multirow[b]{3}{*}{$\mathrm{C}$} & $\mathrm{Mr}$ & $\begin{array}{l}\text { M1(659.456,659.456) } \\
\text { T1(659.456,1318.912) }\end{array}$ & $\begin{array}{l}\text { A2 }(494.592,494.592) \\
\text { P2(494.592,989.184) }\end{array}$ & I & $\begin{array}{l}\text { MR1 }(1648.64,1648.64) \\
\text { MR2 }(1648.64,3297,28)\end{array}$ \\
\hline & ar & $\begin{array}{l}\text { M1 }(1000.56,1000.56) \\
\text { T1 }(1000.56,2001,12)\end{array}$ & $\mathrm{A} 2(1000.56,2001.12) /$ & I & MR1 $(1000.56,2001,12)$ \\
\hline & $\operatorname{tr}$ & $\begin{array}{l}\text { M1(879.795,1173.06) } \\
\text { T1(1173.06,2052.855) }\end{array}$ & $\begin{array}{l}\text { A2 }(1173,06,1173.06) \\
\text { P2(1173.0,2346.12) }\end{array}$ & l & $\begin{array}{c}\text { MR1 }(11773.06,4398.975) \\
\text { MR2 }(879.795,4692.24) \\
\text { MR3 }(879.795 .5572 .03) \\
\text { MR4 }(1173.06,5572.03) \\
\text { MR5 }(43.98 .975,5572.035) \\
\text { MR6 }(4692.24,5572.035)\end{array}$ \\
\hline
\end{tabular}




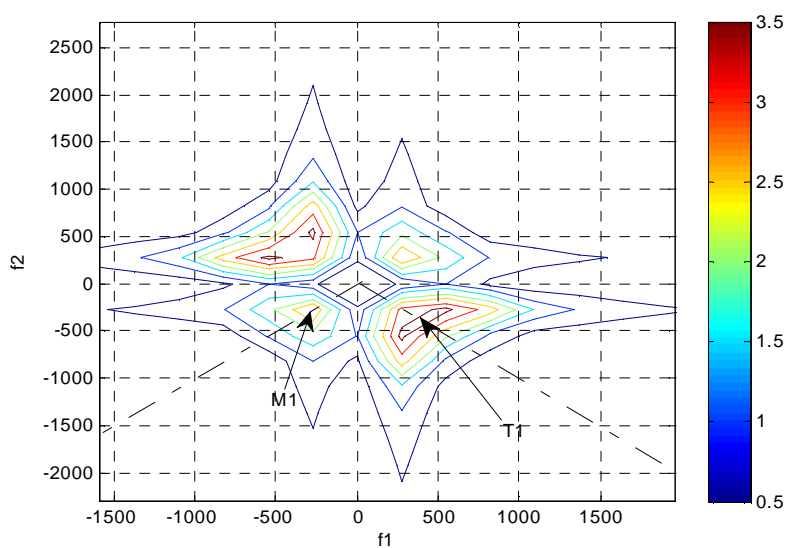

Figure 6. Bispectrum of S1 sound in pathologic case (Group B, record "EC")

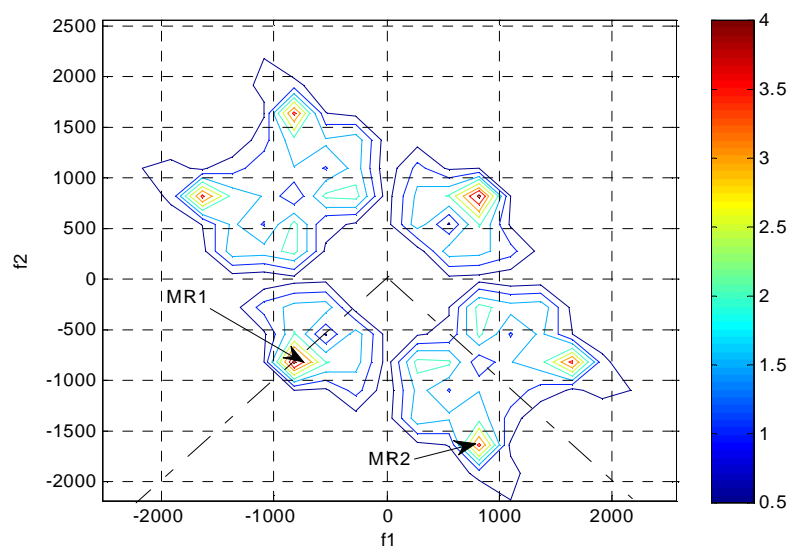

Figure 8. Bispectrum of click sound in pathologic case (Group B, record "EC")

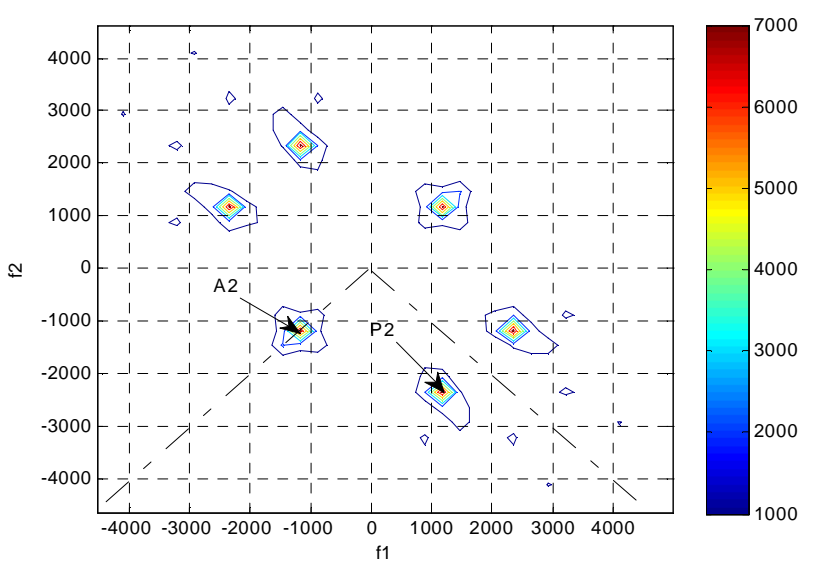

Figure 10. Bispectrum of $\mathrm{S} 2$ sound in pathologic case (Group C, record "mitral-regurgitation")

\section{Group C}

Generally, and as it is illustrated in Table 2, the frequency components in all the records in this group, show that the frequency of $\mathrm{T} 1$ is the double of M1, and P2 is the double of $\mathrm{A} 2$, except in record "tr" (tricuspid-regurgitation), where its bispectra are illustrated in Figures 9 to 11. It shows a predominance of four peaks in the sound S1: (M1, T1, mr1,

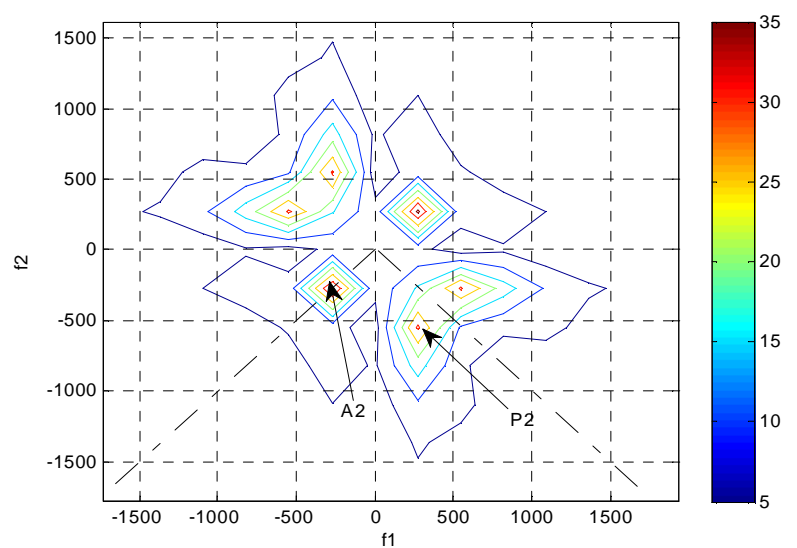

Figure 7. Bispectrum of $\mathrm{S} 2$ sound in pathologic case (Group B, record "EC")

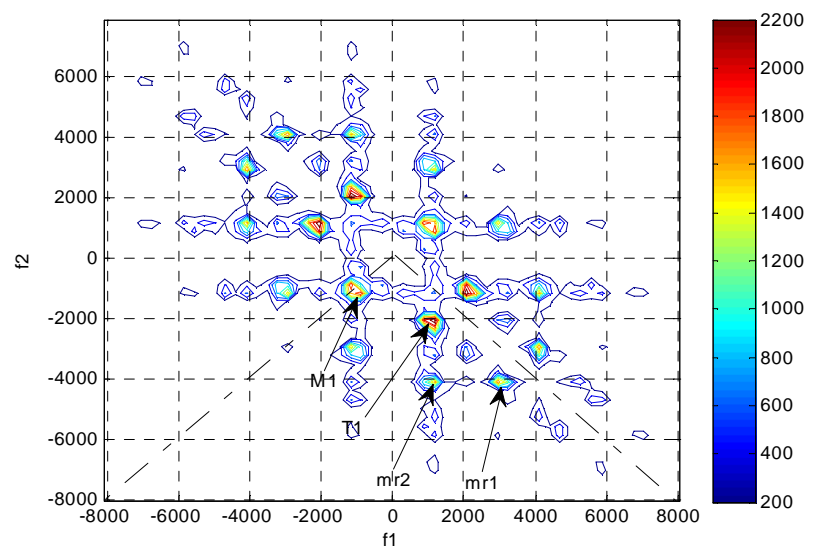

Figure 9. Bispectrum of S1 sound in pathologic case (Group C, record "mitral-regurgitation")

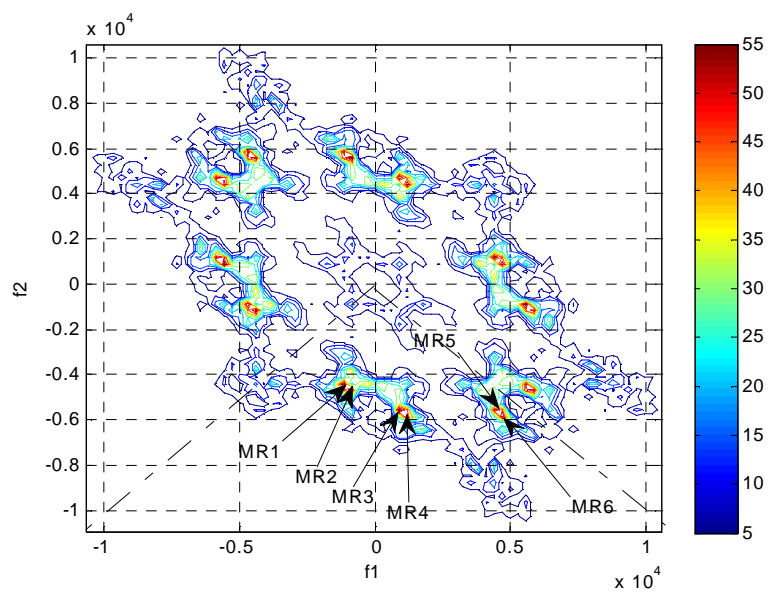

Figure 11. Bispectrum of murmur sound in pathologic case (Group C, record "mitral-regurgitation")

and mr2), two peaks in S2 (A2 and P2), and six peaks (MR1, MR2, MR3, MR4, MR5, and MR6) in murmur sound signal.

$\mathrm{M} 1$ is around $(879.795,1173.06) \mathrm{Hz}, \mathrm{T} 1$ around $(1173.06$, $2052.855) \mathrm{Hz}, \mathrm{mr} 1$ around $(2932.65,405.71) \mathrm{Hz}$, and $\mathrm{mr} 2$ around (1173.06, 4105.71) Hz, (Figure 9).

A2 is around $(1173,06,1173.06) \mathrm{Hz}, \mathrm{P} 2$ around (1173.0, 234612) Hz. (Figure 10). 
Always and as we remark P2 is twice A2. i.e. regardless of the kind of signal: healthy or pathological cases, the frequency of signal corresponding to the closing pulmonic valve is double the frequency of the signal corresponding to the closing aortic valve. However, in this case, T1 is different from M1; because the bispectrum contains two others component ( $\mathrm{mr} 1$ and $\mathrm{mr} 2$ ) corresponding to murmur sound; for the reason that the mitral valve does not close properly and blood surges back to the left atrium during systole, and the murmur start at $\mathrm{S} 1$.

As we remark, the frequencies 2052.855, 2932.65, and $4105.71 \mathrm{~Hz}$ represent a nonlinear relationship between the frequencies $(879.795+1173.06), \quad(2052.855+879.795)$, and $\left(2052.855^{*} 2\right)$, which confirms that $\mathrm{S} 1$ is incorporated in murmur sound signal.

However, the frequencies of the murmur are (Figure 11): MR1 is around (879.795, 5572.35), MR4 is around (1173.06, 5572.035), MR5 is around (43.98.975, 5572.035), and MR6 is around (4692.24, 5572.035).

As we remark there is a nonlinear interaction between the different frequencies: the frequency $5572.035 \mathrm{~Hz}$ is the interaction between 4692.24 and 879.795 (4692.24 +879.795).

The frequency 4398.975 is a nonlinear interaction between the frequency $2052.855 \mathrm{~Hz}$ which was obtained in the bispectrum of S1 and $2346.12 \mathrm{~Hz}$ which was obtained in the bispectrum of S2.

The frequency 4105.71 is the nonlinear interaction between 1173.6 and 2932.65.

On other hand, the frequencies 2932.65 and $2052.855 \mathrm{~Hz}$ represent also harmonic components obtained in S1 bispectrum. However, the frequency 4692.24 is double the frequency $2346.12 \mathrm{~Hz}$ which is obtained in B2 bispectrum.

So we can conclude that the synchronization degree is very high $(5 / 6=83.33 \%)$ (Table 3); because the number of harmonics is five $(5572.035,4398.975,4105.71,2932.65$ and
$2052.855 \mathrm{~Hz}$ ), between six frequencies (879.795, 1173.06, $4692.24,5572.035,4398.975$, and $4105.71 \mathrm{~Hz})$. There is a perfect harmony between frequencies, and might also suggest that one of the obtained frequencies causes the others.

Similar results are obtained in the other records, where we found a high value of synchronization degree, which varies between $60 \%$ and $83.33 \%$ (Table 3).

\section{Evaluation 1}

In this section, we will present a short comparison between, STFT, CWT, and Bispectral analysis. The STFT, WCT analysis algorithms are implemented, and applied on the same used database, but in this paper, we show only the result of one signal as an example.

In the STFT algorithm, the Hanning window was used, with a length of 256, and an overlap of 50\%. However, in the CWT algorithm the wavelet Daubechies 7 (db7) was used.

As it is illustrated in Figure 12, the STFT of the signal "n" shows only one component of each sound (Figure 12b). Each component spreads for large time and large frequency, and can't give us precise information about the number of components and their location in time of each sound.

The obtained results using CWT on the two sounds (S1and S2), are shown in Figure 13a and $\mathbf{b}$. There is high resolution in time and scale compared to STFT results. The CWT shows a predominance of two peaks of each sound (M1 and T1) for the sound $\mathrm{S} 1$ and (A2 and P2) for the sound S2.

It can so easily discriminate between the components of each sound and specifies the location of each of them; however, it remains limited, because it can't give us precise information about the frequencies, or the relationship between them.

Table 3. Synchronization degree

\begin{tabular}{|c|c|c|c|c|c|}
\hline Group & Record & Frequencies of harmonic components & $\begin{array}{c}\text { Number of harmonic } \\
\text { components }\end{array}$ & $\begin{array}{c}\text { Number of the total } \\
\text { frequencies }\end{array}$ & Synchronization degree \\
\hline \multirow{3}{*}{ A } & $\mathrm{n}$ & I & I & I & 0 \\
\hline & coa & I & l & I & 0 \\
\hline & IM & I & l & I & 0 \\
\hline \multirow{5}{*}{$\mathrm{B}$} & $\mathrm{EC}$ & 820.08 & 1 & 4 & $25 \%$ \\
\hline & $\mathrm{AG}$ & 793.17 & 1 & 3 & $1 / 3=33 \%$ \\
\hline & EC1 & $856.605,1142.14,1713.21$ & 3 & 7 & $3 / 7=42 \%$ \\
\hline & MP & 815.715 & 1 & 4 & $1 / 4=25 \%$ \\
\hline & LS & 855.36 & 1 & 3 & $1 / 3=33 \%$ \\
\hline \multirow{3}{*}{$\mathrm{C}$} & $\mathrm{Mr}$ & $1154.048,1813.504,2472.96$ & 3 & 5 & $3 / 5=60 \%$ \\
\hline & ar & $1100616,3101.736,1801.008,2201.232,1300.728$ & 5 & 7 & $5 / 7=71.43 \%$ \\
\hline & $\operatorname{tr}$ & $5572.035,4398.975,4105.71,2932.65,2052.855$ & 5 & 6 & $5 / 6=83.33 \%$ \\
\hline
\end{tabular}


(a)

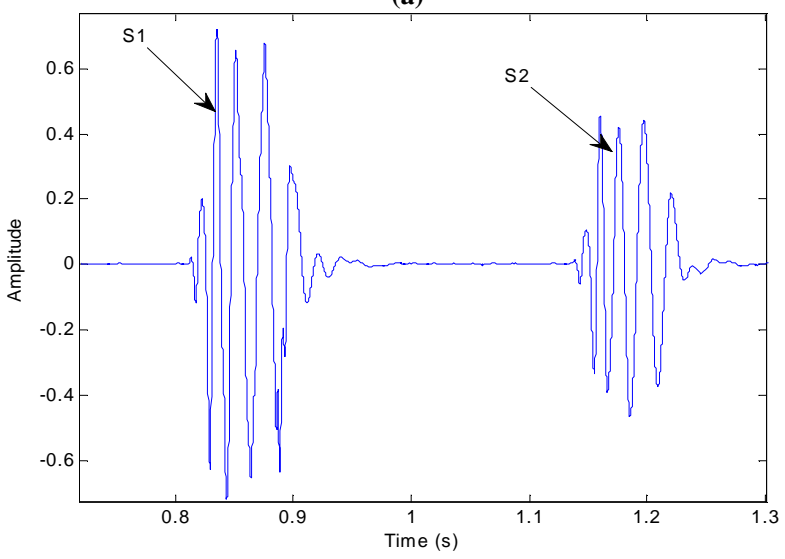

(b)

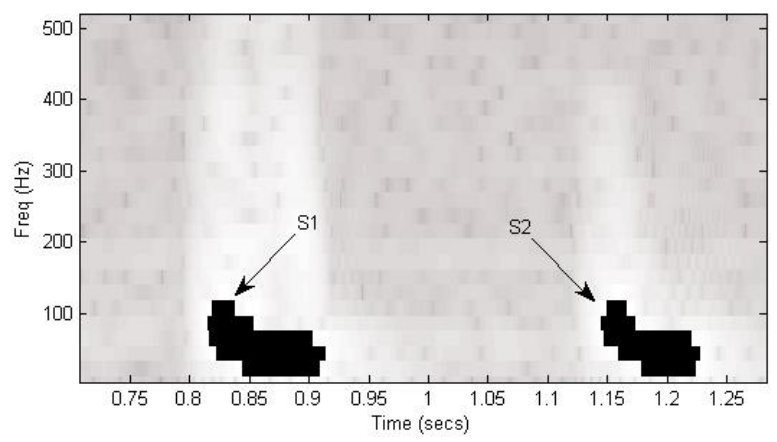

Figure 12. a) Normal PCG signal (one cycle), b) STFT analysis of one cycle of normal PCG signa

(a)

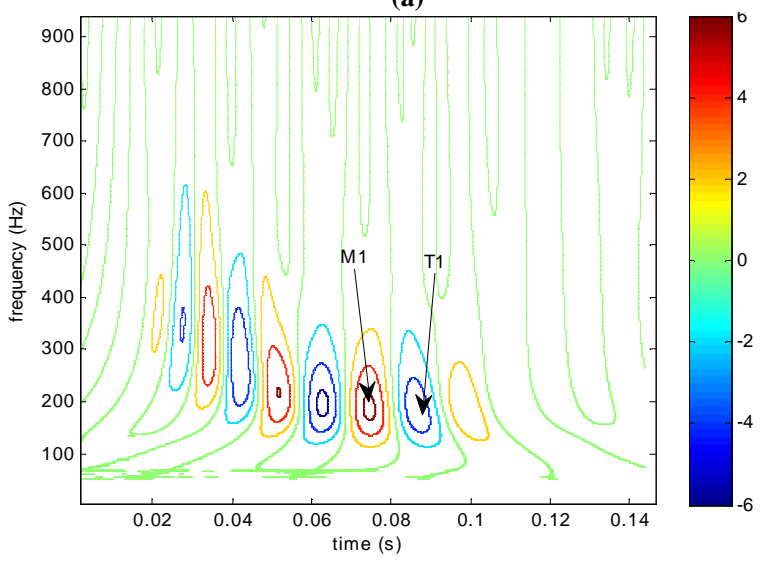

(b)

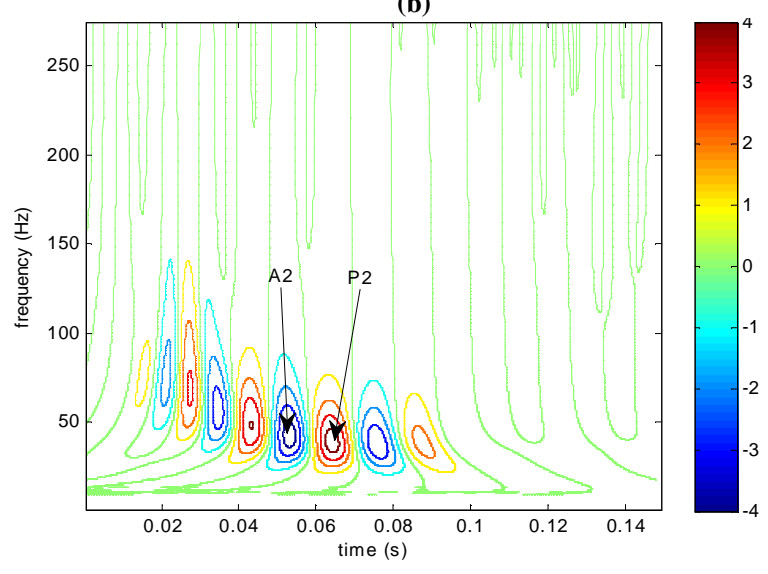

Figure 13. a) CWT analysis of the sound S1, b) CWT analysis of the sound S2

\section{Evaluation 2}

In order to evaluate the proposed algorithm, a comparison between the synchronization degree and energetic ratio is illustrated in Figure 14.

The energetic ratio is an intrinsic characteristic related to the amount of the murmur in one cycle of PCG signal. One of the important indicators of severity is the increased energy of murmur in favor of B1 and B2 sounds.

For this, we use a new parameter, noted RE (energetic ratio), to estimate the degree of severity (Equation 3), which is calculated by the ratio between the energy of the murmur (E2) and the total energy of the signal (one cycle).

The energetic ratio RE is given as a percentage, its maximum value is $100 \%$. This latter will highlight the dominance of the murmur over the B1 and B2 noises. The heart murmurs can be classified according to three degrees of severity: $:^{21}$

light, RE $<30 \%$

Moderate, $30 \%<$ REA $<70 \%$

Severe, $\mathrm{RE}>70 \%$

$R E=\frac{E_{2}}{E_{1}+E_{2}}$

With

$E_{1}$ : Sound energy (B1, B2)

$E_{2}$ : murmur energy
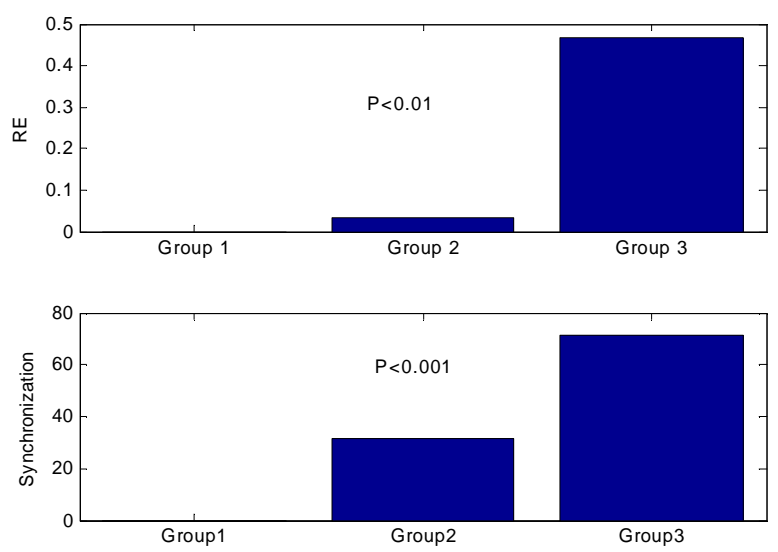

Figure 14. Synchronization and energy ration histogram of the three groups PCG signals

The ANOVA test is a statistical classifier; it is used to identify normal and pathological cases. The p-value is the measure used to identify significant statistical differences between different energetic ratio values. $p<.01$ are considered significant values, while values of $\mathrm{p}<.001$ are considered very significant values.

According to Figure 14, the synchronization degree shows very significant values $(p<.001)$ compared to the energetic ratio $(\mathrm{p}<.01)$, where we found zero values for group A, middle values in group $\mathrm{B}$, and high values in group $\mathrm{C}$. 


\section{Conclusions}

In this paper, an attempt is made to study and analyze the impact of murmurs on cardiac sounds $\mathrm{S} 1$ and $\mathrm{S} 2$ and vice versa using bispectral analysis.

The bispectral analysis is found to be the most successful technique for the analysis of the relationship between sounds S1, S2, clicks, and murmurs on one hand, and between internal components of these sounds on the other hand. The proposed algorithm allows the best representation of this relationship between the different sounds and the internal components (M1, T1, A2, P2, and MRi).

This study shows that the frequencies of sounds S1, S2, clicks, and murmurs are not accidentally generated; but it is generated by the same generator system, and that whatever the pathological severity degree, the heart works in perfect harmony. Even though the STFT can provide correctly the time, but it can't distinguish between the internal components, which are successfully determined by CWT, but it can't find the relationship between them.

We can conclude that the three algorithms (STFT, CWT, and bispectral analysis) are complementary to facilitate a good approach and to better understand the cardiac sounds.

The synchronization degree shows high values in group $\mathrm{C}$, low values for group $\mathrm{B}$, and zero in group $\mathrm{A}$; indicating high severity degrees in signals with murmurs, low severity degrees in signals with clicks, and no danger in signals without clicks or murmurs.

The proposed algorithm, when compared to the energetic ratio showed good discrimination between the three cases according to the ANOVA test.

\section{References}

1. Tilkian A, Conover M. Enderstanding heart sounds and murmurs: with an introduction to lung sounds, 4th edn. Philadelphia, Saunders, 2001.

2. Debbal SD, Bereksi-Reguig F. Complementary analysis to heart sounds while using the short time fourier and the continuous wavelet transforms. Biomedical Engineering: Applications, Basis and Communications. 2007;19(5):331-339. https://doi.org/10.4015/S1016237207000434/

3. Stagmo ML, Persson J. Cardiac and Cardiovascular Systems, Lund University Publications. 2008, ISBN: 978-91-44-01989-5

4. Novey D, Pencar M, Stang, J. The guide to heart sounds: Normal and abnormal, CRC Press Inc, Florida,1990.

5. Shaver J, Salerni R, Reddy P. Normal and abnormal heart sound in cardiac diagnosis. Part I: Systolic sounds. Curr Probl Cardiol;10(3):2-68, 1995. https://doi.org/10.1016/0146-2806(85)90007-6

6. Leung T, White P, Cook J, et al. Analysis of the second heart sound for diagnosis of pediatric heart diseases, IEEE Proc Sci Meas Technol. 1998:145(6):285-290. https://doi.org/10.1049/ip-smt:19982326

7. Yaseen, Son GY, Kwon S. Classification of Heart Sound Signal Using Multiple Features. Appl Sci. 2018;8(12):2344. https://doi.org/10.3390/app8122344

8. Boussa M, Atouf I, Atibi M, et al. Comparison of MFCC and DWT features extractors applied to PCG classification, 11th International Conference on Intelligent Systems: Theories and Applications (SITA), 2016. https://doi.org/10.1109/SITA.2016.7772312

9. Rosół M, Więckowski Ł. Embedded heart rate analysis based on sound sensing, 24th International Conference on Methods and Models in Automation and Robotics (MMAR), Międzyzdroje, Poland, 2019, 629-633. https://doi.org/10.1109/MMAR.2019.8864661

10. http://www.cardiosource.com/heartsounds. Accessed April 25, 2018.

11. American College of Cardiology. http://www.Egeneral medical.com. Accessed November 25, 2006.

12. Heart Sounds and Murmurs. http://www.dundee.ac.uk/medther/Cardiology/hsmur.html. Accessed November 5, 2006.

13. Omari.T, Debbal SM. Etude de degré de sévérité pathologique des sténoses aortiques, magister thesis, Tlemcen University, 2009.

14. Ahmad TJ, Ali H, Khan SA. Classification of Phonocardiogram using an Adaptive Fuzzy Inference System. Proceedings of International Conference on Image Processing, Computer Vision (IPCV), Monte Carlo Resort, Las Vegas, Nevada, USA, 2009: 609$614,2009$.

15. Meziani F, Debbal SM, Atbi A. Analyse du Degré de Sévérité Pathologique de La sténose aortiques(AS) par Application de La transformée en Ondelettes Continue (TOC), a l'occasion de: International Conférence on MultiMedia Information Procession: CMIP'2012, Algérie. 2012

16. Meziani F, Debbal SM, Atbi A. Analysis of the pathological severity degree of aortic stenosis (AS) and mitral stenosis (MS) using the discrete wavelet transform (DWT). Journal of Medical Engineering \& Technology. 2013;37(1):61-74. https://doi.org/10.3109/03091902.2012.733058

17. Chua KC, Chandran V, Acharya UR, Lim CM. Cardiac state diagnosis using higher order spectra of heart rate variability. Journal of Medical Engineering \& Technology. 2008;32(2):145-155. https://doi.org/10.1080/03091900601050862 
18. Goshvarpour A, Goshvarpour A, Rahati S, Saadatian V. Bispectrum estimation of electroencephalogram signal during meditation. Iran J Psychiatry Behav Sci. 2012;6(2):48-54.

19. Nikias C, Raghuveer M. Bispectrum estimation: a digital signal processing framework. Proc IEEE. 1987;75(7):869-91. https://doi.org/10.1109/PROC.1987.13824

20. Chua KC, Chandran V, Acharya R, Lim CM. Higher Order Spectral (HOS) Analysis Of Epileptic EEG Signals, Engineering in Medicine and Biology Society. Proceedings of the 29th Annual International Conference of the IEEE EMBS Cité Internationale, Lyon, France August 23-26, 2007:6495- 6498. https://doi.org/10.1109/IEMBS.2007.4353847

21. Debbal S, Bereksi-Reguig F. Analyse spectro-temporelle des bruits cardiaques par les transformees discrete et continue d'ondelettes. Sciences \& Technologie. B, Sciences De l'ingénieur. 2005(23):5-15. 